PRIMI RISULTATI SULLA PRESENZA E LA

CARATTERIZZAZIONE BIOMOLECOLARE DI NEMATODI ANISAKIDI IN SPECIE ITTICHE MARINE PESCATE NEL NORD SARDEGNA

\title{
FIRST RESULTS ON THE PRESENCE AND THE MOLECULAR CHARACTERIZATION OF ANISAKID NEMATODES IN MARINE FISH CAUGHT OFF NORTHERN SARDINIA
}

\author{
Tedde T. ${ }^{1}$, Piras M.C. ${ }^{2}$, Pinna C. ${ }^{3}$, Virgilio S., Terrosu G. ${ }^{1}$, Piras A. ${ }^{4}$, Garippa G. ${ }^{2}$, Merella P. ${ }^{2}$ \\ ${ }^{1}$ Istituto Zooprofilattico Sperimentale della Sardegna, Sassari; ${ }^{2}$ Università di Sassari, Dipartimento di \\ Biologia Animale, Sezione di Parassitologia e Malattie parassitarie; ${ }^{3}$ Azienda Sanitaria Locale di \\ Sassari, Servizio Veterinario Igiene Alimenti O.A.; ${ }^{4}$ Azienda Sanitaria Locale di Olbia, Servizio \\ Veterinario Igiene Alimenti O.A.
}

\section{SUMMARY}

KEYWORDS

\begin{abstract}
Anisakidosis is a parasitic zoonosis caused by nematodes of the family Anisakidae, belonging to the genera Anisakis, Contracaecum and Pseudoterranova. Molecular studies have shown that Anisakis larvae comprise a number of sibling species, which have different genetic structures, hosts and geographical distribution. A great variety of fish species can harbour infectious third stage larvae of this nematode. The preliminary results of a study carried out to evaluate the occurrence of this parasite in commercial fish caught off northern Sardinia are herein reported. From October 2008 to November 2009, 599 specimens of 8 commercial fish species were examined for anisakid larvae through visual inspection of body cavity and peptic digestion of the muscle. Isolated Anisakis sp. larvae were observed at light microscope and identified as Type I or Type II (sensu Berland, 1961). Out of 599 fish examined, 239 (40\%) were infected by 1187 anisakid larvae, belonging to the genera Anisakis (1169 Type I and 18 Type II) and Hysterothylacium (692). The molecular identification of Anisakis spp. was carried out on a subsample of $30 \%$ of Type I larvae and all Type II larvae. Specimens were firstly examined using a species-specific PCR, with primers designed for Anisakis pegreffii (APEF) and Anisakis physeteris (APHF), and ITS-2 of nuclear rDNA. The results were confirmed by the analysis of the ITS region of nuclear rDNA (ITS-1, 5.8S and ITS-2) using the restriction enzymes HinfI and HhaI in PCR-RFLP. Type I larvae examined were all identified as $A$. pegreffii, and Type II were all A. physeteris. This is the first contribution to the epidemiology and molecular characterization of Anisakis spp. in commercial fish caught off Sardinia.
\end{abstract}

parasitic zoonosis, commercial fish, Anisakis pegreffii, Anisakis physeteris, PCRRFLP.

\section{INTRODUZIONE}

L'anisakidosi è una zoonosi parassitaria causata da larve di nematodi appartenenti a diversi generi della famiglia Anisakidae, fra cui Anisakis, Contracaecum e Pseudoterranova (1).
Tali parassiti hanno un ciclo biologico complesso, nel quale gli ospiti definitivi sono rappresentati da cetacei, pinnipedi ed uccelli ittiofagi, gli ospiti intermedi da piccoli crostacei quali copepodi ed eufausiacei, mentre una grande varietà di ospiti invertebrati e 
vertebrati, tra cui i pesci, fungono da ospiti paparatenici (2). Le specie ittiche commerciali in cui larve del terzo stadio (L3) di Anisakis sp. sono state repertate sono estremamente numerose. La presenza di L3 di Anisakis del tipo I (sensu Berland, 1961, 3) è stata documentata in almeno 200 specie di pesci e 25 di cefalopodi $(4,5)$. Gli stadi larvali di Anisakis sp. rappresentano un potenziale rischio per la salute umana in seguito al consumo di pesci marini o molluschi cefalopodi crudi o poco cotti, oppure sottoposti a procedimenti inadeguati alla loro devitalizzazione, quali salagione, marinatura leggera ed affumicatura a freddo. L'anisakidosi si manifesta nell'uomo in forme cliniche differenti legate alla localizzazione delle larve ed alle relative lesioni anatomopatologiche. Nella forma luminale, asintomatica, le larve non penetrano la mucosa e la diagnosi viene effettuata con il ritrovamento delle larve espulse con tosse, vomito o tramite le feci. Nella forma invasiva le larve penetrano la mucosa gastrica o intestinale e la diagnosi viene effettuata con esame istopatologico o attraverso esame radiologico o gastroscopico. Inoltre, negli ultimi anni è sempre più frequente il riscontro di forme allergiche causate da Anisakis, con una sintomatologia che varia da una semplice orticaria allo shock anafilattico (6). Nel corso degli ultimi 30 anni è stato segnalato un aumento della prevalenza di anisakidosi in diversi paesi. Tale situazione è il risultato dell'interazione di alcuni fattori, quali: miglioramento delle tecniche diagnostiche, incremento della domanda globale di prodotti ittici a base di pesce crudo, marinato o poco cotto (sushi, sashimi, alici, carpaccio, etc), aumento della popolazione di alcuni mammiferi marini che fungono da ospiti definitivi del nematode per l'intensificazione della loro tutela $(6,7)$. In particolare, nell'ultimo decennio sono stati registrati circa 20.000 casi di anisakiasi umana, di cui oltre il $90 \%$ in Giappone (2.000 casi all'anno) e circa il 3,5\% in Europa, soprattutto in Olanda, Germania, Francia e Spagna (8). In Italia sono stati segnalati 23 casi tra il 1996 e il 2007, sempre correlati al consumo di pesce crudo o marinato $(9,10)$.

Scopo del presente lavoro è acquisire informazioni aggiornate relative alla diffusione di larve di nematodi Anisakidae in pesci marini pescati nelle zone costiere del Nord Sardegna (Golfo dell'Asinara), concentrando in particolare l'attenzione su alcune specie ittiche commerciali presenti in diversi habitat e di effettuare una caratterizzazione biomolecolare delle larve di terzo stadio isolate per l'identificazione delle specie di appartenenza.

\section{MATERIALI E METODI}

Tra ottobre 2008 e novembre 2009 sono stati esaminati complessivamente 599 campioni appartenenti a 8 specie di teleostei (3 demersali e 5 pelagiche), di cui 96 Merluccius merluccius, 57 Micromesistius poutassou, 46 Phycis blennoides, 252 Sardina pilchardus, 30 Sardinella aurita, 29 Scomber japonicus, 52 Trachurus mediterraneus, 37 Trachurus trachurus. I campioni esaminati sono stati prelevati da partite di pescato locale proveniente dal Golfo dell'Asinara, presenti nel circuito commerciale. Dopo l'identificazione della specie ittica, gli ospiti sono stati misurati, pesati e sottoposti ad esame parassitologico diretto mediante esame visivo ed osservazione allo stereomicroscopio, seguito da digestione enzimatica del tessuto muscolare (11). I nematodi repertati sono stati montati in alcol glicerinato per l'osservazione al microscopio ottico e quindi identificati in base alle specifiche chiavi morfometriche. Le larve del genere Anisakis sono state differenziate in larve di Tipo I e Tipo II (3). La prevalenza e l'intensità media (12) di ciascun tipo di larva sono state calcolate per ogni specie ittica.

Le larve del terzo stadio di Anisakis sp. isolate sono state conservate in etanolo $70 \%$, ed una parte di queste è stata sottoposta ad analisi biomolecolare per l'identificazione della specie. Le larve sono state accuratamente lavate in acqua distillata sterile e frammentate con l'ausilio di un pestello; per l'estrazione degli acidi nucleici sono stati impiegati appositi kit commerciali (Promega, Qiagen). In una prima fase è stata eseguita un'analisi di PCR speciespecifica per Anisakis pegreffii ed Anisakis physeteris, che rappresentano le specie più diffuse nel Mar Mediterraneo per quanto riguarda le larve di Tipo I e II, rispettivamente (13). In particolare, è stata eseguita l'amplificazione di una porzione della regione genomica nucleare ITS con l'utilizzo dei primers forward APEF

(5'TCTATTTGACATTGTTATTTC-3') per A. pegreffii $\mathrm{e}$ APHF (5'CCTCTGTTTTGACTCATTCGT-3') per A. physeteris, e del primer reverse NC2 (5'TTAGTTTCTTCCTCCGCT -3') per entrambe le specie (14). In una seconda fase è stata effettuata una ulteriore identificazione di specie delle larve del genere Anisakis mediante PCRRFLP con l'utilizzo dell'enzima di restrizione HinfI in accordo con i markers genetici definiti da D'Amelio et al. (15) e implementati da Pontes et al. (16), e dell'enzima HhaI secondo le 
indicazioni di Fang et al. (14). In particolare, è stata eseguita una reazione di amplificazione, mediante PCR, della regione ITS dell' rDNA nucleare (ITS-1, 5.8S e ITS-2) e gli amplificati ottenuti sono stati sottoposti a digestione enzimatica. I prodotti di digestione sono stati rivelati tramite elettroforesi su gel di agarosio al $2 \%$, aggiungendo un controllo positivo e il marker dei pesi molecolari.

\section{RISULTATI}

In $239(40 \%)$ dei 599 pesci esaminati è stata riscontrata la presenza di larve di nematodi Anisakidae appartenenti ai generi Anisakis (1169 del Tipo I e 18 del Tipo II) ed Hysterothylacium (692). In particolare, 146 ospiti $(61,1 \%)$ sono risultati positivi per la sola presenza di L3 di Anisakis sp. e 55 (23,0\%) per la sola presenza di Hysterothylacium sp. In 38 $(15,9 \%)$ degli esemplari esaminati si è osservata una coinfestazione sostenuta da entrambi i generi. Tra gli individui che presentavano larve di Anisakis sp., la percentuale di quelli infestati dal solo Tipo I è risultata pari al $95,1 \%$, la percentuale dal solo Tipo II del 1,1\%, mentre il $3,8 \%$ dei soggetti presentava infestazione mista. Relativamente alla presenza di larve di Anisakis di Tipo I e II negli ospiti esaminati, i valori di prevalenza $(\mathrm{P} \%)$ ed intensità media (I med) più elevati sono stati riscontrati nelle specie T. trachurus, S. japonicus e M. poutassou (Tab. 1).

Tabella 1. Prevalenza (P\%), intensità media (I med) e intervallo di intensità (Interv I) osservati nelle infestazioni da Anisakis Tipo I e Tipo II per specie ittica esaminata.

\begin{tabular}{|ll|rrrr|rrr|}
\hline & & \multicolumn{4}{c|}{ Anisakis Tipo I } & \multicolumn{3}{c|}{ Anisakis Tipo II } \\
\hline \multicolumn{1}{|c|}{ Habitat } & \multicolumn{1}{c|}{ Ospite } & N & P\% & I med & Interv I & P\% & I med & Interv I \\
\hline Demersale & M. merluccius & 96 & $37,5 \%$ & 1,33 & $1-3$ & $0,0 \%$ & - & - \\
Demersale & M. poutassou & 57 & $61,4 \%$ & 3,90 & $1-50$ & $5,3 \%$ & 1,00 & $1-3$ \\
Demersale & P. blennoides & 46 & $0,0 \%$ & - & - & $0,0 \%$ & - & - \\
Pelagico & S. pilchardus & 252 & $13,1 \%$ & 1,20 & $1-3$ & $0,0 \%$ & - & - \\
Pelagico & S. aurita & 30 & $13,3 \%$ & 1,00 & $1-1$ & $0,0 \%$ & - & - \\
Pelagico & S. japonicus & 29 & $96,6 \%$ & 15,10 & $1-46$ & $3,4 \%$ & 1,00 & $1-1$ \\
Pelagico & T. mediterraneus & 52 & $11,5 \%$ & 1,50 & $1-3$ & $0,0 \%$ & - & - \\
Pelagico & T. trachurus & 37 & $100,0 \%$ & 13,65 & $1-98$ & $5,4 \%$ & 2,00 & $1-2$ \\
\hline
\end{tabular}

Su un totale di 184 soggetti infestati da L3 di Anisakis sp., $40(21,7 \%)$ sono risultati positivi sia all'esame visivo che alla digestione enzimatica e $144(78,2 \%)$ solo all'esame visivo. In particolare nel muscolo sono state esclusivamente repertate larve di Anisakis del Tipo I, la cui prevalenza nelle diverse specie ittiche esaminate è risultata pari a $37,8 \%$ per $T$. trachurus, $20,7 \%$ S. japonicus, $10,5 \% \quad M$. poutassou, $6,7 \%$ S. aurita, 5,2\% M. merluccius e $2,8 \%$ per $S$. pilchardus.

Figura 1: PCR specie-specifica Lanes: M Molecular weigth marker VIII; 1-6 A. pegreffi; 8-13 A. physeteris; 7,14 Controlli negative

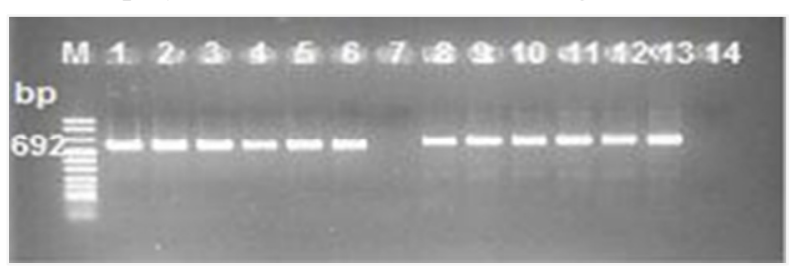

Per quanto riguarda la caratterizzazione biomolecolare, l'utilizzo di primers speciespecifici ha consentito di identificare come $A$. pegreffii tutte le larve del Tipo I sino ad ora esaminate (351), con un prodotto di amplificazione di $577 \mathrm{bp}$ e come $A$. physeteris tutte le larve del Tipo II, con un amplificato di 637 bp (Fig. 1). Successivamente, l'applicazione della metodica PCR-RFLP ha confermato i risultati ottenuti.

Figura 2: RFLP patterns della regione ITS con HinfI e HhaI. Lanes: M Molecular weigth marker VIII; 1 Uncut; 2-4 A. pegreffi 5-7 A. physeteris; 8-10 A. pegreffi; 11-13 A. physeteris

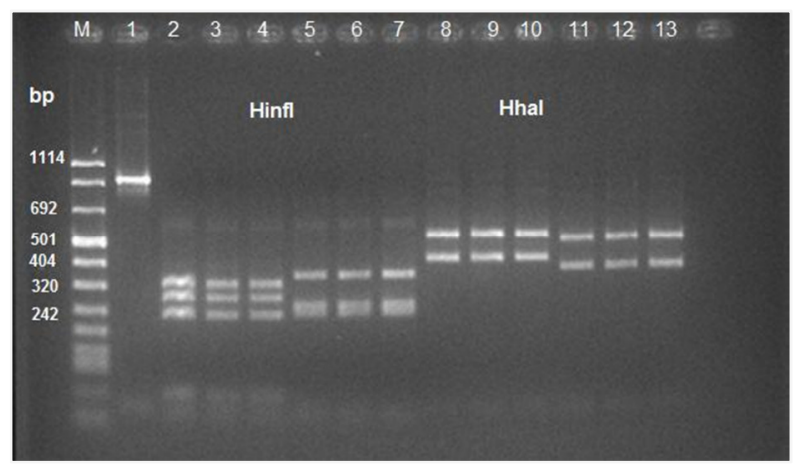

Infatti, in base alla combinazione dei patterns 
RFLP, tutte le forme larvali del Tipo I sono stastate identificate come appartenenti alla specie A. pegreffii e tutte le larve del Tipo II alla specie A. physeteris. In particolare, l'amplificazione della regione di rDNA ITS (ITS-1, 5.8S e ITS-2) ha prodotto un frammento di circa $1000 \mathrm{bp}$, la cui digestione mediante l'enzima Hinf $\mathrm{I}$ ha evidenziato tre bande di 370, 300, 250 bp per $A$. pegreffii e tre bande di 380, 290, 270 bp per $A$. physeteris. La digestione effettuata con l'enzima HhaI ha prodotto due bande di 550 e $450 \mathrm{bp}$ per A. pegreffii, e due bande di 510 e $390 \mathrm{bp}$ per $A$. physeteris (Fig. 2).

\section{CONSIDERAZIONI E CONCLUSIONI}

Il rischio sanitario associato alla presenza di larve di Anisakis sp. nei prodotti ittici è conosciuto da tempo ed è oggetto di attenzione da parte delle attuali normative nazionali (Circ. Min. San. 11/03/1992 n.10, O.M. 12/05/1992) e comunitarie (Reg CE 853/2004, Reg. 2074/2005). L'attualità di tali norme è peraltro pienamente giustificata dai recenti cambiamenti delle abitudini alimentari di fasce sempre più ampie di consumatori che negli ultimi anni apprezzano le preparazioni a base di pesce crudo o poco cotto. Nella tradizione culinaria italiana esistono tuttavia da tempo pietanze a base di prodotti ittici sottoposti a marinatura leggera che sono all'origine del maggior numero di casi di anisakiosi nella penisola (17). Uno dei principali strumenti sui quali è basata l'Analisi del Rischio (Reg. CE 178/2002, art. 6), è rappresentato dall'attivazione di adeguati piani di sorveglianza epidemiologica basati sulla raccolta e documentazione di dati scientificamente sostenibili. In questa ottica, il presente lavoro condotto con l'applicazione di metodiche biomolecolari (PCR specie-specifica, PCR-RFLP), ha consentito di acquisire nuove informazioni epidemiologiche sulla diffusione di nematodi Anisakidae nei prodotti della pesca provenienti da zone costiere del nord Sardegna (Golfo dell'Asinara). Relativamente all'isolamento di larve del genere Anisakis, i risultati ottenuti confermano $i$ valori di prevalenza riscontrati da altri autori $(18,19,20)$ in alcune specie ittiche del mar Mediterraneo (T. trachurus, S. japonicus e M. poutassou) note per la presenza di nematodi infestanti. In particolare, le uniche specie di Anisakis riscontrate sono state $A$. pegreffii per il Tipo I ed A. physeteris per il Tipo II, che sono quelle maggiormente diffuse nel Mar mediterraneo (13).

Per quanto riguarda $S$. pilchardus, contrariamente a quanto riscontrato in esemplari provenienti dalle coste atlantiche e mediterranee della Spagna (21), è stata registrata una prevalenza di larve di Anisakis Tipo I pari al $13,1 \%$, tutte appartenenti alla specie A. pegreffii.

$\mathrm{Si}$ sottolinea inoltre come il frequente isolamento di nematodi del genere Hysterothylacium (23,0\%), considerati non patogeni per l'uomo in quanto gli ospiti definitivi sono rappresentati da pesci predatori (22) e talvolta presenti contemporaneamente negli esemplari infestati da L3 di Anisakis sp., imponga la necessità di una corretta identificazione degli anisakidi riscontrati nei prodotti ittici esaminati. Un contributo importante alla valutazione del rischio sanitario per il consumatore è dato dai risultati relativi alla ricerca di L3 di Anisakis sp. nel muscolo delle diverse specie ittiche esaminate. Questa ha rivelato una maggiore prevalenza dei nematodi nelle specie $T$. trachurus $(37,8 \%), S$. japonicus $(20,7 \%)$ e $M$. poutassou (10,5\%). Il frequente riscontro di tali nematodi nelle parti dei pesci destinate al consumo umano rende necessaria l'adozione di appropriate misure di profilassi in grado di limitare la migrazione nel muscolo delle larve presenti nella cavità celomatica, in particolare una pronta eviscerazione dei pesci subito dopo la cattura. La normativa attualmente in vigore vieta l'immissione sul mercato e la successiva somministrazione di preparazioni a base di pesce crudo che non siano state precedentemente trattate mediante congelamento ad una temperatura pari a $-20^{\circ} \mathrm{C}$ per almeno 24 ore in stabilimenti autorizzati.

Il riscontro di L3 di Anisakis sp. nei prodotti della pesca in Italia è stato più volte oggetto di segnalazioni di allerta che, talvolta, sono apparse ingiustificate in quanto basate sulla presenza di nematodi non vitali esclusivamente all'interno della cavità celomatica dei pesci. A tale proposito infatti, secondo una nota DGSAN a commento delle indicazioni della Commissione Sanco-RASFF, il riscontro di Anisakis nelle parti del pesce non destinate al consumo umano rappresenta un problema di tipo qualitativo. Tuttavia, la Commissione non esclude il pericolo rappresentato dalla possibilità che antigeni di larve di Anisakis devitalizzate possano determinare nel consumatore una reazione allergica dopo l'ingestione (6) e pertanto stabilisce che possano essere accettate le notifiche basate sia sul riscontro di L3 di Anisakis sp. vive o anche devitalizzate nelle parti dei prodotti ittici destinate al consumo, sia sulla presenza di L3 
vive all'interno della cavità celomatica.

\section{BIBLIOGRAFIA}

1. Thomas C.D., Lawrence R.A., (1995). Anisakis and Pseudoterranova spp. In: Parasites in Human Tissues. American Society of Clinical Pathologists Press, Chicago, pp.104-109.

2. Mattiucci S., Nascetti G. (2006). Molecular systematics, phylogeny and ecology of anisakid nematodes of the genus Anisakis Dujardin, 1845: an update. Parasite 13:99113

3. Berland B. (1961) Nematodes from some Norwegian marine fishes. Sarsia 2:1-50

4. Smith J.W. \& Wootten R., 1978. Anisakis and anisakiasis. Advances in Parasitology 16: $93-163$

5. Hochberg F.G., (1990). Diseases of Mollusca: Cephalopoda En: Kinne O (ed). Diseases of marine animals, Vol. III: Introduction, Cephalopoda, Annelida, Crustacea, Chaetognatha, Echinodermata and Urochordata, pp. 47-227. Biologische Anstalt Helgoland, Hamburg.

6. Chai J-Y., Murrell K.D., Lymbery A.J., (2005). Fish-borne parasitic zoonoses: Status and issues. International Journal for Parasitology 35: 1233-1254.

7. Oshima T. (1987). Anisakiasis - Is the Sushi Bar Guilty? Parasitology Today 3: 4448.

8. Abe N. (2008). Application of the PCRsequence-specific primers for the discrimination among larval Anisakis simplex complex. Parasitology Res. 102:1073-1075.

9. Maggi P., Iambrenghi O., Scardigno A., Scoppetta L., Saracino A., Valente M., Pastore G., Angarano G., (2000) Gastrointestinal infection due to Anisakis simplex in southern Italy. European Journal of Epydemiology 16: 75-78.

10. Urgenti I., Lattarulo S., Ferrarese F., De ceglie A., Manta R., Brandonsio O. (2007): Acute gastric anisakiasis: An Italian axperience. Minerva Chirurgica, 62, 51-60.

11. Food and Drug Administration (1984). Parasitic animals in food. In Bacteriological analytical manual $6^{\text {th }}$ ed., Association of Official Analitical Chemists, Arlington, Virginia, pp.21.01-21.09..

12. Bush A.O., Lafferty K.D., Lotz J.M., Shostak A.W., 1997. Parasitology meets ecology on its own terms: Margolis et al. revisited. Journal of Parasitology, 83, 575583.
13. Mattiucci S., Nascetti G., (2006). Molecular systematics, phylogeny and ecology of anisakid nematodes of the genus Anisakis Dujardin, 1845: an update. Parasite 13:99113.

14. Fang W., Xu S., Zhang S., Wang Y., Chen X., Luo D., (2010). Multiple primer PCR for the identification of anisakid nematodes from Taiwan Strait. Experimental Parasitology 124: 197-201

15. D'Amelio S., Mathiopoulos KD., Santos CP., Pugachev ON., Webb SC., Picanco MO., Paggi L., (2000) Genetic markers in ribosomal DNA for the identification of members of the genus Anisakis (Nematoda: Ascarioidea) defined by polymerase chain reaction-based restriction fragment length polymorphism. International Journal Parasitology 30: 223-226.

16. Pontes T., D'Amelio S., Costa G., Paggi L., (2005). Molecular cheracterization of larval anisakid nematodes from marine fishes of Madeira by a PCR-based approach, with evidence for a new species. Journal of Parasitology, 91: 1430-1434.

17. Pampiglione S., Rivasi F., Criscuolo M., De Benettis A., Gentile A., Russo S., Testini M., Villani M. (2002): Human Anisakiasis in Italy: A Report of Eleven New Cases. Pathology Research and Practice 198: 429434.

18. Costa A., Sciortino S., Reale S., Alio V., Cusimano M., Caracappa S. (2008) Indagine sulla presenza di nematodi Anisakidae in specie ittiche marine. Atti $\mathrm{X}$ congresso Nazionale S.I.Di.L.V., Alghero 22-24 Ottobre 2008.

19. Mattiucci S., Abaunza P., Ramadori L., Nascetti G., (2004) Genetic identification of Anisakis larvae in European hake from Atlantic and Mediterranean waters for stock recognition. Journal of Fish Biology 64: 495-510.

20. Farjallah S., Slimane B., Busi M., Paggi L., Amor N., Blel H., Said K., D'Amelio S., (2008). Occurrence and molecular identification of Anisakis spp. From the North African coast of editerranean Sea. Parasitology Res. 102: 371-379

21. Rello F., Adroher J. , Valero A. (2008) Hysterothylacium aduncum, the only anisakid parasite of sardines (Sardina pilchardus) from the southern and eastern coast of Spain. Parasitology Res. 104: 117121.

22. Anderson R.C. (2000): Nematode parasites of vertebrates. Their development and 
A.I.V.I online Giugno 2011, vol. 1 n. 0

transmission. $2^{\text {nd }}$ ed. CABI Publishing,
Wallingford. 